\author{
Kazuma Yamakawa \\ Hiroshi Ogura \\ Satoshi Fujimi \\ Miki Morikawa \\ Yoshihito Ogawa \\ Tomoyoshi Mohri \\ Yasushi Nakamori \\ Yoshiaki Inoue \\ Yasuyuki Kuwagata \\ Hiroshi Tanaka \\ Toshimitsu Hamasaki \\ Takeshi Shimazu
}

\section{Recombinant human soluble thrombomodulin in sepsis-induced disseminated intravascular coagulation: a multicenter propensity score analysis}

Received: 20 August 2012

Accepted: 9 December 2012

Published online: 30 January 2013

(C) The Author(s) 2013. This article is published with open access at

Springerlink.com

K. Yamakawa $(\bowtie) \cdot H$. Ogura •

Y. Ogawa · Y. Kuwagata · T. Shimazu Department of Traumatology and Acute Critical Medicine, Osaka University Graduate School of Medicine, 2-15, Yamadaoka, Suita, Osaka 565-0871, Japan e-mail: k.yamakawa0911@gmail.com

Tel.: +81-6-68795707

Fax: +81-6-68795720

K. Yamakawa $\cdot$ S. Fujimi .

Y. Ogawa · T. Mohri · Y. Nakamori Department of Emergency and Critical Care, Osaka General Medical Center, 3-1-56, Bandai-Higashi, Sumiyoshi-ku, Osaka 558-8558, Japan

M. Morikawa - Y. Inoue $\cdot$ H. Tanaka Department of Emergency and Critical Care Medicine, Juntendo University Urayasu Hospital, 2-1-1, Tomioka, Urayasu,

Chiba 279-0021, Japan

T. Hamasaki

Department of Biomedical Statistics, Osaka University Graduate School of Medicine, 2-15, Yamadaoka, Suita,

Osaka 565-0871, Japan
Abstract Purpose: Evidence of efficacy and safety of, and especially mortality related to, recombinant human thrombomodulin (rhTM) treatment for sepsis-induced disseminated intravascular coagulation (DIC) is limited. We hypothesized that patients with sepsis-induced DIC receiving treatment with rhTM would have improved mortality compared with those with similar acuity who did not. Methods: This retrospective cohort study conducted in three tertiary referral hospitals in Japan between January 2006 and June 2011 included all patients with sepsisinduced DIC who required ventilator management. Primary endpoint was in-hospital mortality, with duration of intensive care unit treatment, changes in DIC scores and rate of bleeding complications as secondary endpoints. Regression technique was used to develop a propensity model adjusted for baseline imbalances between groups. Results: Eligible were 162 patients with sepsis-induced DIC; 68 patients received rhTM and 94 did not. Patients receiving rhTM had higher severity of illness according to baseline characteristics. After adjusting for these imbalances by stratified propensity score analysis, treatment with rhTM was significantly associated with reduced inhospital mortality (adjusted hazard ratio, 0.45 ; $95 \%$ confidential interval, $0.26-0.77 ; p=0.013)$. An association between rhTM treatment and higher numbers of intensive care unit-free days, ventilator-free days, and vasopressor-free days were observed. DIC scores were significantly decreased in the rhTM group compared with the control group in the early period after rhTM treatment, whereas the incidence of bleedingrelated adverse events did not differ between the two groups. Conclusions: Therapy with rhTM may be associated with reduced in-hospital mortality in adult mechanically ventilated patients with sepsis-induced DIC.

Keywords Sepsis · DIC .

Anticoagulant therapy .

Thrombomodulin .

Outcome assessment .

Retrospective studies

\section{Introduction}

Sepsis-induced disseminated intravascular coagulation (DIC) is a life-threatening disease characterized by the systemic activation of blood coagulation that generates intravascular fibrin leading to multiple organ dysfunction syndrome or even death $[1,2]$. A large gap in appraisal of anticoagulant therapies for sepsis-induced DIC is 
observed around the world, and therapy other than supportive care may not be warranted for treating DIC. Although guidelines for the diagnosis and treatment of DIC in Britain [3], Japan [4], and Italy [5] have recently been published, there are some distinct discrepancies regarding levels of recommendation of various anticoagulant therapies among these guidelines. The novel biological agent recombinant human soluble thrombomodulin (rhTM) was approved and is being used clinically for treatment of DIC in Japan, whereas this agent is currently being evaluated in other countries. Currently, the efficacy of rhTM in sepsis-induced DIC remains a matter of dispute because of limited evidence that it improves clinical outcomes.

Several animal studies have demonstrated a reduction in mortality with the administration of rhTM in a severe sepsis model [6,7], whereas clinical studies have been limited. In a phase III randomized controlled trial in Japan, significant improvement in the rate of DIC resolution in the rhTM group was shown in comparison with the heparin group, but the effects of rhTM on mortality were not clarified due to the insufficient number of patients with sepsis-induced DIC [8, 9]. In a previous single-center before-and-after study, we demonstrated that rhTM administration could attenuate organ dysfunctions, especially respiratory dysfunction, in patients with sepsis-induced DIC [10, 11]. The effects of rhTM on mortality, however, have not been thoroughly evaluated. Therefore, the main objective of the present study was to analyze the effect of rhTM treatment for sepsis-induced DIC on in-hospital mortality in a retrospective multicenter study.

\section{Methods}

Study population

This investigation was a retrospective cohort study of patients admitted to the medical-surgical intensive care units (ICUs) in three tertiary referral hospitals in Japan between January 2006 and June 2011. Patients were assigned to one of two groups, those receiving rhTM (rhTM group) and those not receiving rhTM (control group). Inclusion criteria were: a known or suspected infection on the basis of clinical data at study entry, two or more signs of systemic inflammation with at least the presence of sepsis-induced organ dysfunction, hematologic dysfunction (platelet count of $<80,000 / \mathrm{mm}^{3}$ ), and the necessity of mechanical ventilation to stabilize the patient's general condition. All patients fulfilled the criteria of the Japanese Association for Acute Medicine (JAAM) DIC scoring system [12]. Exclusion criteria were as follows: fatal or life-threatening bleeding (intracranial, gastrointestinal, or pulmonary bleeding); history of cerebrovascular disorder (cerebral bleeding, cerebral infarction) within 1 year; age $\leq 15$ years; history of hypersensitivity to protein preparations or unfractionated heparin; pregnancy or breast-feeding; and fulminant hepatitis, decompensated liver cirrhosis, or other serious liver disorder.

All patients were principally treated according to the strategy of the Surviving Sepsis Campaign Guidelines $[13,14]$. We did not administer recombinant human activated protein $\mathrm{C}$ (rhAPC) in either group because the use of rhAPC has not been approved for the treatment of severe sepsis in Japan. In our retrospective study, there was no predefined protocol regarding the definite indications for rhTM treatment. For patients with severe sepsis fulfilling the criteria of DIC, rhTM was used at the discretion of the attending physician. In the rhTM group, rhTM was principally administered intravenously at the dose of $0.06 \mathrm{mg} / \mathrm{kg}$ per day, and the infusion was continued for 6 days. Administration of antithrombin concentrate or heparin was not routinely performed, but in the few cases in which either agent was administered, it was done so at the discretion of the attending physician based on individual patient condition.

This study followed the principles of the Declaration of Helsinki, and the conduction of this study was approved by the institutional review board at Osaka General Medical Center. The board waived the need for informed consent for a retrospective study such as this study.

\section{Data collection}

Baseline characteristics including demographic information and information on preexisting conditions, organ function/failure, infection, and pertinent medications were collected. The variables considered to assess comparability among the two groups were age, sex, Acute Physiology and Chronic Health Evaluation (APACHE) II score, Sequential Organ Failure Assessment (SOFA) score, number of dysfunctional organs, site of infection, and rate of positive blood culture.

The primary outcome measure was in-hospital mortality. We also evaluated 28-, 60-, and 90-day and ICU mortality from all causes. ICU-specific outcomes included length of ICU stay, number of days on mechanical ventilation, and number of days spent on vasopressors, each evaluated respectively as number of ICU-free days, number of vasopressor-free days, and number of ventilator-free days. For example, we evaluated ventilator-free days as the number of days to day 28 that the patient achieved unassisted breathing, assuming that the patient survived to 28 days and did not require assisted breathing. Patients who did not survive to 28 days were assigned zero ventilator-free days. ICU-free and vasopressor-free days were evaluated in a similar manner.

Both the JAAM and the International Society of Thrombosis and Hemostasis (ISTH) DIC scores were 
recorded on the day the patient met the inclusion criteria for baseline values and on days 3 and 7 after study entry. Scoring systems for the JAAM DIC definitions are found elsewhere [12]. The ISTH overt DIC definitions were adopted as proposed by Taylor et al. [15] for platelet counts, prothrombin time, and fibrinogen level. Fibrin/ fibrinogen degradation products values were chosen as the fibrin-related marker and scored according to the cut-off levels and ranges recently published by Gando et al. [16]

We also recorded complications, including the occurrence of intracranial hemorrhage, gastrointestinal hemorrhage, respiratory tract hemorrhage, and minor transfusions. Minor transfusion was defined as the administration of four or more units of red blood cells within two consecutive days up to 14 days.

\section{Statistical analysis}

Due to the retrospective nature of the study, baseline imbalances between patients with and without rhTM therapy existed; therefore, an adjusted mortality analysis was performed using propensity scores [17, 18]. Baseline patient variables used as candidates in the modeling process were clinically and statistically considered as confounders that conceivably could bear on the choice to administer rhTM. The propensity score for receiving rhTM was calculated by using multivariate logistic regression and included 15 independent variables comprising age, sex, severity of illness (APACHE II score, SOFA score, number of dysfunctional organs, and positive blood culture), JAAM DIC score, respiratory dysfunction, time from severe sepsis onset to study entry (within $48 \mathrm{~h}$ or not), severe conditions in the past medical history (diabetes mellitus and immunosuppressive therapy), site of infection, and therapeutic interventions (heparin administration, antithrombin administration, emergency operation). The $c$ statistic was 0.792 . The Hosmer-Lemeshow chi-square value was $9.585(d f=8)$, with a nonsignificant $p$ value of 0.295 , which indicates that the model is well-fitted.

Adjustment for the probability of receiving treatment was accomplished by a stratified method. This approach allowed full use of the data and also stratum-by-stratum estimates of the rhTM hazard ratio (HR). Patients were stratified into quintiles according to their propensity scores. The overall effectiveness of treatment on mortality was assessed by propensity score-stratified log-rank test. In addition, adjusted curves of time to mortality and HR with its $95 \%$ confidence interval (CI) were estimated by a Cox regression model with the strata by propensity score. For secondary ICU-specific outcomes of ICU-free days, ventilator-free days, and vasopressor-free days, regression analyses for censored non-normal data with propensity score were performed because the data were censored and not normally distributed. For secondary outcomes of bleeding complications, propensity-adjusted CochranMantel-Haenszel analyses were performed. Odds ratio (OR) and its $95 \%$ CI were estimated by logistic regression with the strata by propensity score.

A marginal structural model was also used to assess the robustness of the conclusions from the stratified method, and no major significant differences between the methods were found. An alternative to the propensity score techniques used in this study (stratification, regression adjustment, and weighting) is to select a sample of patients comparable in each treatment group (propensity score matching). However, we did not use propensity score matching because of several issues with this method in relation to unmatched exclusion, as discussed in Kurth et al. [19].

Changes of coagulation parameters from baseline levels were analyzed with the use of a mixed random effects model adjusted for the baseline values as a covariate, and by post hoc Tukey test. For patients with missing data, we used the last-observation-carried-forward method of imputation [20]. Missing data occurred because of death, discharge from hospital, and samples not drawn. In addition, to assess the robustness of the results from the last-observation-carried-forward method, complete case analysis was also performed and supported the results.

Descriptive statistics are presented as the mean (95\% $\mathrm{CI})$, median (interquartile range), or number (percent), as appropriate. Univariate differences between groups were assessed using the Mann-Whitney $U$ test and chi-square test or Fisher's exact test, as appropriate. A $p$ value of $<0.05$ was considered to indicate statistical significance. All statistical analyses were performed with SAS Statistical Software version 9.1.3 (SAS Institute Inc., Cary, NC, USA).

\section{Results}

Baseline characteristics

The patient flow diagram is shown in Fig. 1. During the study period, 162 consecutive patients fulfilled the above inclusion criteria. The rhTM group comprised 68 patients, and the control group comprised 94 patients. Baseline characteristics and therapeutic interventions of the study population are shown in Table 1 . The baseline characteristic of severity of illness, as indicated by APACHE II and SOFA scores, number of dysfunctional organs, and rate of positive blood culture, was significantly higher in the rhTM group than in the control group. There were no significant differences in coagulation parameters including DIC scores, severe conditions in the past medical history, site of infection, or therapeutic interventions between the two groups. 


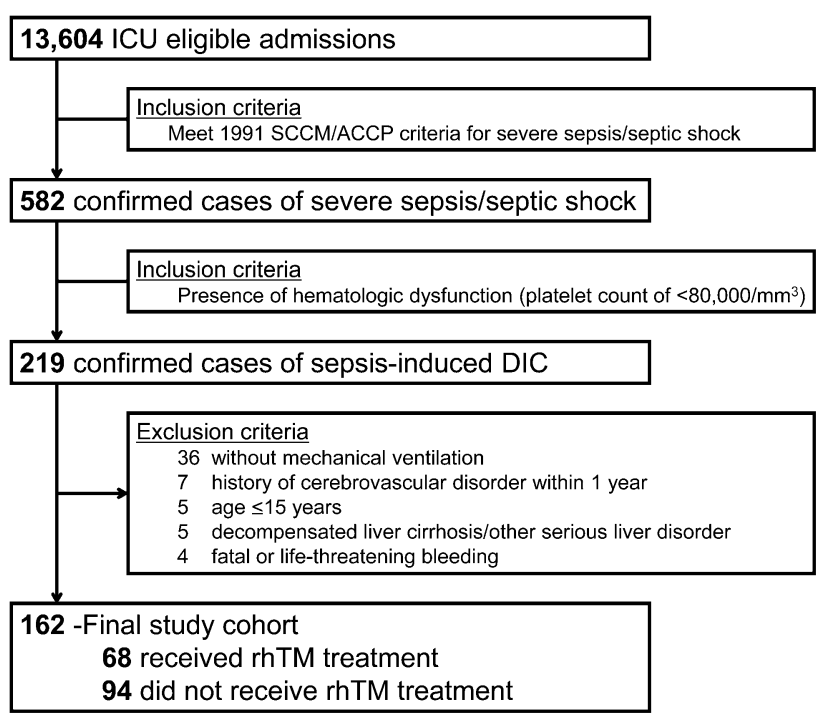

Fig. 1 Patient flow diagram. ICU intensive care unit, SCCM Society of Critical Care Medicine, ACCP American College of Chest Physicians, DIC disseminated intravascular coagulation, rhTM recombinant human soluble thrombomodulin

The median time from onset of severe sepsis to study entry was similar in the two groups (1.0 [interquartile range 0.0-2.0] days in the rhTM group vs. 1.0 [0.0-2.0] days in the control group, $p=0.199$ ). For the rhTM group, the median duration of rhTM administration was 6.0 (interquartile range 4.0-6.0) days, and the median dose of rhTM administration was 0.059 (0.043-0.065) $\mathrm{mg} / \mathrm{kg} / \mathrm{day}$.

\section{Effect of treatment on mortality}

Main outcomes relating to mortality are presented in Table 2, and the survival curves of the prediction model by covariates of propensity score calculated by Cox regression analysis over the 90-day study period are given for both the rhTM and control groups in Fig. 2. In a stratified propensity score analysis, receipt of rhTM was associated with reduced in-hospital mortality (adjusted $\mathrm{HR}, 0.45 ; 95 \%$ CI $0.26-0.77 ; p=0.013$ ). In addition, favorable significance in the rhTM group in comparison with the control group was also shown for mortality at 28, 60 , and 90 days, and for ICU mortality $(p=0.017$, $p=0.006, p=0.003$, and $p=0.018$, respectively, by stratified log-rank test adjusted by propensity score).

\section{Effect of treatment on ICU duration}

An association between rhTM treatment and longer period of ICU treatment-free days was observed in analyses stratified by quintile of propensity score (Table 3 ).
Patients treated with rhTM had a longer period of adjusted median ICU-free days within 28 days (10.8 [95 \% CI 8.9-12.4] days in the rhTM group vs. 8.0 [5.7-9.7] days in the control group, $p=0.040$ ). In addition, favorable significance of the rhTM group in number of ventilator-free days (15.0 [13.2-16.6] vs. 10.7 [8.6-12.5] days, $p=0.002$ ) and vasopressor-free days (20.1 [18.1-21.9] vs. 14.4 [12.1-16.4] days, $p<0.001)$ was shown.

\section{Effect of treatment on DIC parameters}

In the rhTM group, both JAAM and ISTH DIC scores were significantly decreased compared with the control group on day 3 , in the early period after rhTM administration (Fig. 3a, b). Although there was no difference in platelet counts between the two groups on day 3, the recovery of platelet counts in the rhTM group on day 7 after rhTM treatment was greater than that in the control group, as shown in Fig. 3c.

\section{Adverse events}

Bleeding complications during the study period are presented in Table 4. Rates of gastrointestinal bleeding (7.4 \% in the rhTM group vs. $6.4 \%$ in the control group, $p=0.598)$, respiratory tract bleeding ( 0 vs. $4.3 \%$, $p=0.768)$, and intracranial bleeding (1.5 vs. $1.1 \%$, $p=0.568$ ) were similar in the treated and untreated groups. After adjustment for the strata by propensity score, the percentage of patients who received minor transfusion was also similar in the two groups (33.8 vs. $50.0 \%, p=0.108)$.

\section{Discussion}

The results of this study provide evidence that rhTM administration improved mortality and shortened the time on mechanical ventilation, time on vasopressors, and length of ICU stay of adult patients with sepsis-induced DIC. We also found that both JAAM and ISTH DIC scores were rapidly normalized by the rhTM treatment. We previously investigated the efficacy of rhTM in single-institution studies, and the results from this multicenter study will increase the reliability of the evidence of the efficacy of rhTM.

rhAPC has been focused on as a treatment option for DIC complicating sepsis [3,5] because this drug was shown to improve mortality in the PROWESS (Protein C Worldwide Evaluation in Severe Sepsis) trial 10 years ago $[21,22]$. However, in response to the failure of the 
Table 1 Baseline characteristics of patients with sepsis-induced disseminated intravascular coagulation treated or not treated with rhTM

\begin{tabular}{|c|c|c|c|c|}
\hline & Overall $(n=162)$ & $\operatorname{rhTM}$ group $(n=68)$ & Control group $(n=94)$ & $p$ value $^{\mathrm{a}}$ \\
\hline \multicolumn{5}{|l|}{ Patient characteristics } \\
\hline Age, years ${ }^{\mathrm{b}}$ & $69(59-76)$ & $69(61-76)$ & $70(57-77)$ & 0.953 \\
\hline Male sex ${ }^{b}$ & $93(57)$ & $36(53)$ & $57(61)$ & 0.339 \\
\hline \multicolumn{5}{|l|}{ Severity of illness } \\
\hline APACHE II score ${ }^{b}$ & $23(19-29)$ & $25(21-32)$ & $22(18-27)$ & 0.008 \\
\hline SOFA score ${ }^{\mathrm{b}}$ & $11(9-13)$ & $12(9-13)$ & $11(8-12)$ & 0.029 \\
\hline Number of dysfunctional organs ${ }^{b}$ & $4(3-5)$ & $4(3-5)$ & $4(3-5)$ & 0.383 \\
\hline Positive blood culture ${ }^{\mathrm{b}}$ & $72(44)$ & $41(60)$ & $31(33)$ & 0.001 \\
\hline \multicolumn{5}{|l|}{ Coagulation parameters } \\
\hline Platelet count, /mm & $4.9(2.7-6.5)$ & $4.4(2.6-6.4)$ & $5.3(2.8-6.6)$ & 0.081 \\
\hline PT-INR & $1.40(1.23-1.70)$ & $1.40(1.20-1.67)$ & $1.50(1.30-1.78)$ & 0.169 \\
\hline $\mathrm{FDP}, \mu \mathrm{g} / \mathrm{mL}$ & $22.3(11.0-55.5)$ & $24.6(13.2-60.0)$ & $20.3(10.2-48.9)$ & 0.380 \\
\hline Fibrinogen level, mg/dL & $350(224-495)$ & $357(225-553)$ & $328(213-456)$ & 0.231 \\
\hline JAAM DIC score ${ }^{\mathrm{b}}$ & $6(5-8)$ & $6(5-8)$ & $6(5-8)$ & 0.555 \\
\hline ISTH DIC score & $4(3-5)$ & $4(4-5)$ & $4(3-5)$ & 0.457 \\
\hline \multicolumn{5}{|l|}{ Organ failure } \\
\hline Respiratory ${ }^{\mathrm{b}}$ & $114(70)$ & $54(79)$ & $60(64)$ & 0.037 \\
\hline Circulatory & $134(83)$ & $57(84)$ & $77(82)$ & 0.835 \\
\hline Kidney & $86(53)$ & $37(54)$ & $49(52)$ & 0.873 \\
\hline Metabolic & $96(59)$ & $40(59)$ & $56(60)$ & 1.000 \\
\hline Hematologic & $162(100)$ & $68(100)$ & $94(100)$ & 1.000 \\
\hline Time from severe sepsis onset to study entry ${ }^{\mathrm{b}}$ & & & & 0.689 \\
\hline Early $(<48 \mathrm{~h})$ & $114(70)$ & $49(72)$ & $65(69)$ & \\
\hline Late $(>48 \mathrm{~h})$ & $48(30)$ & $19(28)$ & $29(31)$ & \\
\hline \multicolumn{5}{|l|}{ Co-morbidities } \\
\hline Diabetes $^{\mathrm{b}}$ & $32(20)$ & $17(25)$ & $15(16)$ & 0.167 \\
\hline Hypertension & $38(24)$ & $18(27)$ & $20(21)$ & 0.458 \\
\hline Hemodialysis & $9(6)$ & $2(3)$ & $7(7)$ & 0.306 \\
\hline Immunosuppression $^{\mathrm{b}}$ & $19(12)$ & $11(16)$ & $8(9)$ & 0.146 \\
\hline Malignant Disease & $12(7)$ & $5(7)$ & $7(7)$ & 1.000 \\
\hline Site of infection ${ }^{\mathrm{b}}$ & & & & $0.100^{\mathrm{c}}$ \\
\hline Lung & $32(20)$ & $10(15)$ & $22(23)$ & \\
\hline Abdomen & $62(38)$ & $23(34)$ & $39(42)$ & \\
\hline Soft tissue & 31 (19) & 13 (19) & 18 (19) & \\
\hline Urinary tract & $21(13)$ & $14(21)$ & $7(7)$ & \\
\hline Other/unknown & $16(10)$ & 8 (12) & $8(9)$ & \\
\hline \multicolumn{5}{|l|}{ Therapeutic interventions } \\
\hline Vasopressor & $131(81)$ & $57(84)$ & $74(79)$ & 0.429 \\
\hline Steroid & $49(30)$ & $16(24)$ & $33(35)$ & 0.123 \\
\hline Heparin/heparinoid ${ }^{\mathrm{b}}$ & $18(11)$ & $4(6)$ & $14(15)$ & 0.081 \\
\hline Antithrombin ${ }^{\mathrm{b}}$ & $16(10)$ & $9(13)$ & $7(7)$ & 0.288 \\
\hline Renal replacement therapy & $48(30)$ & $20(29)$ & $28(30)$ & 1.000 \\
\hline Emergency operation $^{\mathrm{b}}$ & $63(39)$ & $20(29)$ & $43(46)$ & 0.050 \\
\hline
\end{tabular}

Data are expressed as group median (interquartile range) or number intravascular coagulation, ISTH International Society on Throm(percent)

rhTM recombinant human soluble thrombomodulin, APACHE Acute Physiology and Chronic Health Evaluation, SOFA Sequential Organ Failure Assessment, $P T$-INR prothrombin time-international normalized ratio, FDP fibrin/fibrinogen degradation products, JAAM Japanese Association for Acute Medicine, DIC disseminated bosis and Haemostasis

${ }^{a} p$ value for rhTM group vs. control group

b The 15 variables used in propensity score calculation

c Only one $p$ value, for site of infection, is shown because the test is being done as a chi-square test on a $2 \times 5$ crosstable

PROWESS-SHOCK trial to demonstrate efficacy of rhAPC [23], the drug was withdrawn from the worldwide market [24]. As a result, at this stage we have no alternative anticoagulant therapy for sepsis-induced DIC that has been proven with high-quality evidence. Administration of antithrombin concentrate is also recognized for its ability to improve DIC, but it is not used in clinical medicine due to its inability to improve mortality in patients with severe sepsis [25]. Although a recommendation level for the

administration of rhTM was first described in the Italian guidelines, administration of rhTM was not recommended in DIC patients due to the lack of high-quality research to verify improvement of mortality [5]. The present study is the first report, to our knowledge, in which mortality was defined as a primary outcome associated with the effects of rhTM in patients with sepsis-induced DIC.

In this study, we also investigated the length of time spent in critical care. A significant increase in the number 
Table 2 Main outcomes of patients with sepsis-induced disseminated intravascular coagulation treated or not treated with rhTM

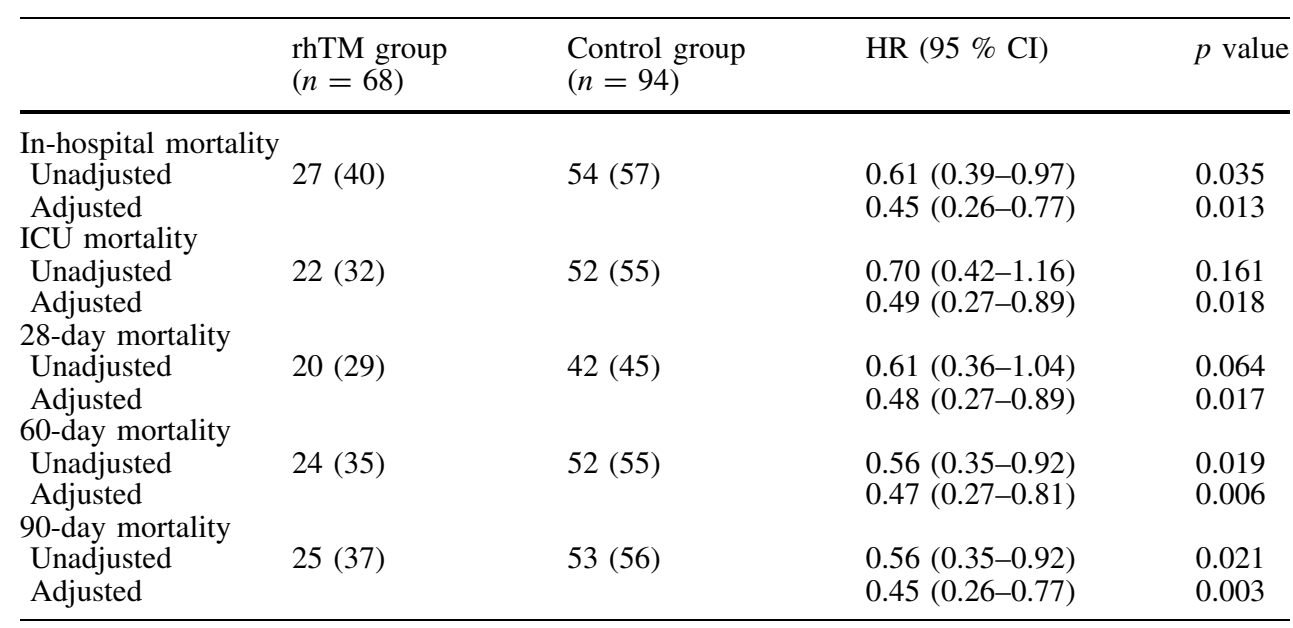

Data are expressed as number (percent). The overall effectiveness of treatment on mortality was assessed by propensity score-stratified log-rank test. Hazard ratio (HR) with its $95 \%$ confidence interval (CI) was estimated by Cox regression model with the strata by propensity score. A propensity model for receiving recombinant human soluble thrombomodulin (rhTM) was built using multivariate logistic regression and included 15 independent baseline variables (see "Methods" section for details)

ICU intensive care unit

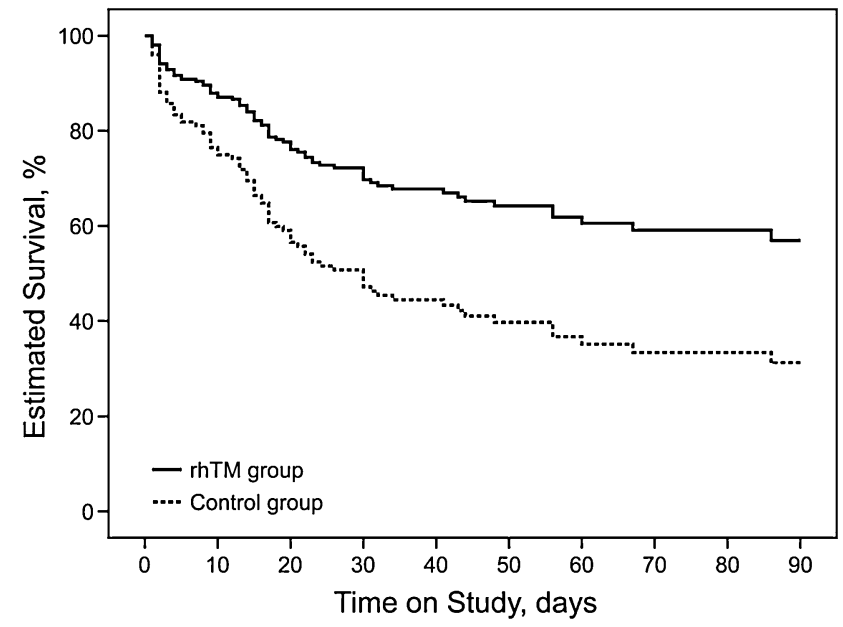

Fig. 2 Adjusted estimated survival curves by covariates of propensity score in Cox regression models. The solid line represents patients in the rhTM group, and the dotted line represents patients in the control group. A propensity model for receiving rhTM was built using multivariate logistic regression and included 15 independent baseline variables (see "Methods" section for details). Treatment with rhTM was associated with a significantly higher rate of survival $(p=0.003)$. $r h T M$ recombinant human soluble thrombomodulin

of ventilator-free days and vasopressor-free days was demonstrated in the rhTM group compared with the control group. Consequently, the number of ICU-free days was also increased in the rhTM group. In the pathological progress of severe sepsis, inflammatory activation in patients with severe sepsis is almost invariably related to the production of inflammatory mediators but also to the activation of coagulation. The coagulation system is a major participant in acute lung injury, and activation of coagulation is considered to be both a consequence of and contributor to ongoing lung injury. Increased thrombin results in diffuse alveolar fibrin deposition, which serves to amplify pulmonary inflammation [26]. It is clear from animal studies that rhTM prevents lipopolysaccharide-induced pulmonary injury by inhibiting pulmonary accumulation of leukocytes [27]. We previously reported that rhTM improves respiratory dysfunction in patients with severe sepsis, as supported by the decreased respiratory component of the SOFA and lung injury scores [11], and total SOFA score in the rhTM group also rapidly decreased compared with that in the control group [10]. As a result of these early beneficial effects on organ dysfunction, rhTM may improve mortality in mechanically ventilated patients with sepsisinduced DIC.

Increased risk of bleeding is the greatest concern with rhTM administration, as well as with rhAPC [28]. However, rhTM is thought to have some favorable effects on the reduction of bleeding complications in comparison with rhAPC. Because rhTM exerts its anticoagulant effect in a thrombin-dependent manner, rhTM does not activate protein $\mathrm{C}$ after inhibiting thrombin generation. In the present study, we showed that hemorrhagic complications, such as gastrointestinal, respiratory tract, or intracranial bleeding, were not increased by rhTM administration. In the phase III trial of rhTM in Japan, the incidence of bleeding complications was lower in the rhTM group than in the heparin group [8], which is compatible with the observations in the present study.

Being retrospective, our study has some limitations. First, the adoption of a nonrandomized design brings its 
Table 3 ICU-specific outcomes of patients with sepsis-induced disseminated intravascular coagulation treated or not treated with rhTM

\begin{tabular}{llll}
\hline & $\begin{array}{l}\text { rhTM group } \\
(n=68)\end{array}$ & $\begin{array}{l}\text { Control group } \\
(n=94)\end{array}$ & $p$ value \\
\hline $\begin{array}{l}\text { Ventilator-free days } \\
\text { Unadjusted }\end{array}$ & $14.8(13.1-16.2)$ & $11.0(9.0-12.6)$ & 0.002 \\
$\begin{array}{l}\text { Adjusted } \\
\text { Vasopressor-free days }\end{array}$ & $15.0(13.2-16.6)$ & $10.7(8.6-12.5)$ & 0.002 \\
$\begin{array}{l}\text { Unadjusted } \\
\text { Adjusted }\end{array}$ & $19.7(17.8-21.3)$ & $14.9(12.7-16.7)$ & $<0.001$ \\
$\begin{array}{l}\text { ICU-free days } \\
\text { Unadjusted }\end{array}$ & $20.1(18.1-21.9)$ & $14.4(12.1-16.4)$ & $<0.001$ \\
Adjusted & $10.9(9.2-12.4)$ & & 0.012 \\
\hline
\end{tabular}

Data are expressed as group means (95\% confidence intervals). Effects of treatment on intensive care unit (ICU) duration were analyzed using regression analyses for censored non-normal data with propensity score. A propensity model for receiving recombinant human soluble thrombomodulin (rhTM) was built using multivariate logistic regression and included 15 independent baseline variables (see "Methods" section for details)
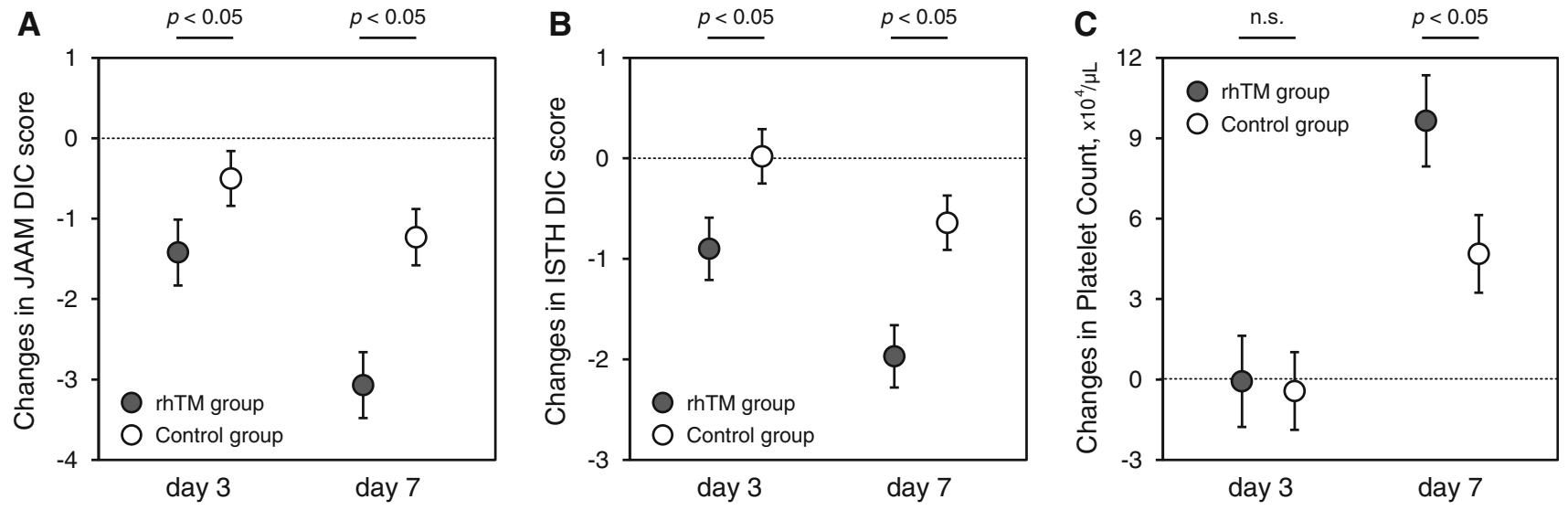

Fig. 3 Changes from baseline in JAAM DIC score (a), ISTH DIC effects model adjusted for the baseline values as a covariate and by score (b) and platelet counts (c). Data are expressed as group adjusted least square means with $95 \%$ confidence interval. The closed circles represent patients in the rhTM group, and the open circles represent patients in the control group. Changes from baseline levels were analyzed with the use of the mixed random post hoc Tukey test. rhTM recombinant human soluble thrombomodulin, JAAM Japanese Association for Acute Medicine, DIC disseminated intravascular coagulation, ISTH International Society on Thrombosis and Haemostasis

Table 4 Bleeding complications of patients with sepsis-induced disseminated intravascular coagulation treated or not treated with rhTM

\begin{tabular}{|c|c|c|c|c|}
\hline & $\begin{array}{l}\text { rhTM group } \\
(n=68)\end{array}$ & $\begin{array}{l}\text { Control group } \\
(n=94)\end{array}$ & OR $(95 \% \mathrm{CI})$ & $p$ value \\
\hline \multicolumn{5}{|c|}{ Gastrointestinal bleeding } \\
\hline $\begin{array}{l}\text { Unadjusted } \\
\text { Adjusted }\end{array}$ & $5(7)$ & $6(6)$ & $\begin{array}{l}1.16(0.34-3.98) \\
1.43(0.38-5.43)\end{array}$ & $\begin{array}{l}0.809 \\
0.598\end{array}$ \\
\hline \multicolumn{5}{|c|}{ Respiratory tract bleeding } \\
\hline $\begin{array}{l}\text { Unadjusted } \\
\text { Adjusted }\end{array}$ & $0(0)$ & $4(4)$ & $\begin{array}{l}0.26(0.00-2.08) \\
0.68(0.00-5.23)\end{array}$ & $\begin{array}{l}0.221 \\
0.768\end{array}$ \\
\hline \multicolumn{5}{|c|}{ Intracranial bleeding } \\
\hline $\begin{array}{l}\text { Unadjusted } \\
\text { Adjusted }\end{array}$ & $1(2)$ & $1(1)$ & $\begin{array}{l}1.39(0.09-22.59) \\
0.45(0.03-7.48)\end{array}$ & $\begin{array}{l}0.817 \\
0.568\end{array}$ \\
\hline $\begin{array}{l}\text { Any transfusi } \\
\text { Unadjusted } \\
\text { Adjusted }\end{array}$ & $23(34)$ & $47(50)$ & $\begin{array}{l}0.51(0.27-0.97) \\
0.55(0.26-1.15)\end{array}$ & $\begin{array}{l}0.040 \\
0.108\end{array}$ \\
\hline
\end{tabular}

Data are expressed as number (percent). Rate of bleeding complications were analyzed using propensity-adjusted Cochran-Mantel-Haenszel analyses. Odds ratio (OR) and its $95 \%$ confidence interval $(\mathrm{CI})$ were estimated by logistic regression with the strata by propensity score. A propensity model for receiving recombinant human soluble thrombomodulin (rhTM) was built using multivariate logistic regression and included 15 independent baseline variables (see "Methods" section for details) 
share of methodological issues. Nevertheless, a careful and rigorous analysis can lead to adequate estimates. We developed a propensity score approach to cope with nonrandomization. The propensity score methods are easy to implement and force the analysts to explicitly focus on recruitment biases. Second, the relatively long time span of the study could have been associated with the introduction of therapeutic measures able to influence the outcome independently from rhTM. Third, a small number of patients were included in this study compared with the other large trial. The combination of these three limitations might cause multiple unmeasured variables to account for the outcome differences observed in this study. Also, we recognize that the design of the present study is not adequate to address the safety of the treatment. Further multicenter prospective randomized trials would be needed to thoroughly evaluate the effects and safety of rhTM on treatment of sepsis-induced DIC.

In conclusion, we found that rhTM may have a significant beneficial effect on mortality in patients with sepsis-induced DIC. In addition, time on mechanical ventilation, time on vasopressors, and length of ICU stay were shorter in the rhTM group than in the control group, whereas bleeding complications did not differ significantly between the two groups. Further clinical investigations are necessary to evaluate the effect of rhTM on the pathophysiology of sepsis-induced DIC.

Acknowledgments No financial support was received in the performance of this study.

Conflicts of interest Drs. Yamakawa and Inoue have received lecture honoraria from Asahi Kasei Pharma Corporation. The remaining authors have not disclosed any potential conflicts of interest.

Open Access This article is distributed under the terms of the Creative Commons Attribution Noncommercial License which permits any noncommercial use, distribution, and reproduction in any medium, provided the original author(s) and the source are credited.

\section{References}

1. Levi M, van der Poll T (2010) Inflammation and coagulation. Crit Care Med 38:S26-S34

2. Esmon CT (2005) The interactions between inflammation and coagulation. Br J Haematol 131:417-430

3. Levi M, Toh CH, Thachil J, Watson HG (2009) Guidelines for the diagnosis and management of disseminated intravascular coagulation. British Committee for Standards in Haematology. Br J Haematol 145:24-33

4. Wada H, Asakura H, Okamoto K et al (2010) Expert consensus for the treatment of disseminated intravascular coagulation in Japan. Thromb Res 125:6-11

5. Di Nisio M, Baudo F, Cosmi B, D'Angelo A, De Gasperi A, Malato A, Schiavoni M, Squizzato A, Italian Society for Thrombosis and Haemostasis (2012) Diagnosis and treatment of disseminated intravascular coagulation: guidelines of the Italian Society for Haemostasis and Thrombosis (SISET). Thromb Res 129:e177-e184

6. Nagato M, Okamoto K, Abe Y, Higure A, Yamaguchi K (2009) Recombinant human soluble thrombomodulin decreases the plasma high-mobility group box-1 protein levels, whereas improving the acute liver injury and survival rates in experimental endotoxemia. Crit Care Med $37: 2181-2186$
7. Iba T, Nakarai E, Takayama T, Nakajima K, Sasaoka T, Ohno Y (2009) Combination effect of antithrombin and recombinant human soluble thrombomodulin in a lipopolysaccharide induced rat sepsis model. Crit Care 13:R203

8. Saito H, Maruyama I, Shimazaki S, Yamamoto Y, Aikawa N, Ohno R, Hirayama A, Matsuda T, Asakura H, Nakashima M, Aoki N (2007) Efficacy and safety of recombinant human soluble thrombomodulin (ART-123) in disseminated intravascular coagulation: results of a phase III, randomized, double-blind clinical trial. J Thromb Haemost 5:31-41

9. Aikawa N, Shimazaki S, Yamamoto Y, Saito H, Maruyama I, Ohno R,

Hirayama A, Aoki Y, Aoki N (2011) Thrombomodulin alfa in the treatment of infectious patients complicated by disseminated intravascular coagulation: subanalysis from the phase 3 trial. Shock 35:349-354

10. Yamakawa K, Fujimi S, Mohri T, Matsuda H, Nakamori Y, Hirose T, Tasaki O, Ogura H, Kuwagata Y, Hamasaki T, Shimazu T (2011) Treatment effects of recombinant human soluble thrombomodulin in patients with severe sepsis: a historical control study. Crit Care 15:R123
11. Ogawa Y, Yamakawa K, Ogura H, Kiguchi T, Mohri T, Nakamori Y, Kuwagata Y, Shimazu T, Hamasaki T, Fujimi S (2012) Recombinant human soluble thrombomodulin improves mortality and respiratory dysfunction in patients with severe sepsis. J Trauma Acute Care Surg 72:1150-1157

12. Gando S, Iba T, Eguchi Y et al (2006) A multicenter, prospective validation of disseminated intravascular coagulation diagnostic criteria for critically ill patients: comparing current criteria. Crit Care Med 34:625-631

13. Dellinger RP, Carlet JM, Masur $\mathrm{H}$ et al (2004) Surviving Sepsis Campaign guidelines for management of severe sepsis and septic shock. Intensiv Care Med 30:536-555

14. Dellinger RP, Levy MM, Carlet JM et al (2008) Surviving Sepsis Campaign: international guidelines for management of severe sepsis and septic shock: 2008. Intensiv Care Med 34:17-60

15. Taylor FB Jr, Toh CH, Hoots WK, Wada H, Levi M, Scientific Subcommittee on Disseminated Intravascular Coagulation (DIC) of the International Society on Thrombosis and Haemostasis (ISTH) (2001) Towards definition, clinical and laboratory criteria, and a scoring system for disseminated intravascular coagulation. Thromb Haemost 86:1327-1330 
16. Gando S, Saitoh D, Ogura $\mathrm{H}$ et al (2008) Natural history of disseminated intravascular coagulation diagnosed based on the newly established diagnostic criteria for critically ill patients: results of a multicenter, prospective survey. Crit Care Med 36:145-150

17. D'Agostino RB Jr, D'Agostino RB Sr (2007) Estimating treatment effects using observational data. JAMA 297:314-316

18. Gayat E, Pirracchio R, Resche-Rigon M, Mebazaa A, Mary JY, Porcher R (2010) Propensity scores in intensive care and anaesthesiology literature: a systematic review. Intensiv Care Med 36:1993-2003

19. Kurth T, Walker AM, Glynn RJ, Chan KA, Gaziano JM, Berger K, Robins JM (2006) Results of multivariable logistic regression, propensity matching, propensity adjustment, and propensitybased weighting under conditions of nonuniform effect. Am J Epidemiol 163:262-270
20. Cheng B, Chow S (2003) Validity of LOCF. In: Chow $S$ (ed) Encyclopedia of biopharmaceutical statistics, 2nd edn. Inf Healthc, London, pp 1023-1029

21. Bernard GR, Vincent JL, Laterre PF et al (2001) Efficacy and safety of recombinant human activated protein $\mathrm{C}$ for severe sepsis. N Engl J Med 344:699-709

22. Dhainaut JF, Yan SB, Joyce DE, Pettilä V, Basson B, Brandt JT, Sundin DP, Levi M (2004) Treatment effects of drotrecogin alfa (activated) in patients with severe sepsis with or without overt disseminated intravascular coagulation. J Thromb Haemost 2:1924-1933

23. Ranieri VM, Thompson BT, Barie PS et al (2012) Drotrecogin alfa (activated) in adults with septic shock. N Engl J Med 366:2055-2064
24. Angus DC (2012) Drotrecogin alfa (activated)... a sad final fizzle to a roller-coaster party. Crit Care 16:107

25. Warren BL, Eid A, Singer P et al (2001) Caring for the critically ill patient. High-dose antithrombin III in severe sepsis: a randomized controlled trial. JAMA 286:1869-1878

26. Finigan JH (2009) The coagulation system and pulmonary endothelial function in acute lung injury. Microvasc Res 77:35-38

27. Uchiba M, Okajima K, Murakami K, Johno M, Okabe H, Takatsuki K (1996) Recombinant thrombomodulin prevents endotoxin-induced lung injury in rats by inhibiting leukocyte activation. Am J Physiol 271:L470-L475

28. Martí-Carvajal AJ, Solà I, Lathyris D, Cardona AF (2012) Human recombinant activated protein $\mathrm{C}$ for severe sepsis. Cochrane Database Syst Rev 3:CD004388 